\title{
Bilateral vestibulopathy and age: experimental considerations for testing dynamic visual acuity on a treadmill
}

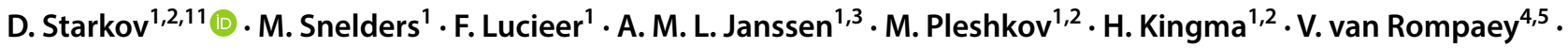 \\ N. Herssens ${ }^{6,7} \cdot$ A. Hallemans $s^{6,7,8} \cdot$ L. Vereeck $^{6,7} \cdot$ C. McCrum $^{9} \cdot$ K. Meijer $^{9} \cdot$ N. Guinand ${ }^{1,10} \cdot$ A. Perez-Fornos ${ }^{10}$. \\ R. van de Berg ${ }^{1,2}$
}

Received: 20 February 2020 / Revised: 25 May 2020 / Accepted: 28 September 2020 / Published online: 28 October 2020

(c) The Author(s) 2020

\begin{abstract}
Introduction Bilateral vestibulopathy (BVP) can affect visual acuity in dynamic conditions, like walking. This can be assessed by testing Dynamic Visual Acuity (DVA) on a treadmill at different walking speeds. Apart from BVP, age itself might influence DVA and the ability to complete the test. The objective of this study was to investigate whether DVA tested while walking, and the drop-out rate (the inability to complete all walking speeds of the test) are significantly influenced by age in BVP-patients and healthy subjects.

Methods Forty-four BVP-patients (20 male, mean age 59 years) and 63 healthy subjects ( 27 male, mean age 46 years) performed the DVA test on a treadmill at 0 (static condition), 2, 4 and $6 \mathrm{~km} / \mathrm{h}$ (dynamic conditions). The dynamic visual acuity loss was calculated as the difference between visual acuity in the static condition and visual acuity in each walking condition. The dependency of the drop-out rate and dynamic visual acuity loss on BVP and age was investigated at all walking speeds, as well as the dependency of dynamic visual acuity loss on speed.

Results Age and BVP significantly increased the drop-out rate $(p \leq 0.038)$. A significantly higher dynamic visual acuity loss was found at all speeds in BVP-patients compared to healthy subjects $(p<0.001)$. Age showed no effect on dynamic visual acuity loss in both groups. In BVP-patients, increasing walking speeds resulted in higher dynamic visual acuity loss $(p \leq 0.036)$.

Conclusion DVA tested while walking on a treadmill, is one of the few "close to reality" functional outcome measures of vestibular function in the vertical plane. It is able to demonstrate significant loss of DVA in bilateral vestibulopathy patients. However, since bilateral vestibulopathy and age significantly increase the drop-out rate at faster walking speeds, it is recommended to use age-matched controls. Furthermore, it could be considered to use an individual "preferred" walking speed and to limit maximum walking speed in older subjects when testing DVA on a treadmill.
\end{abstract}

Keywords Dynamic visual acuity $\cdot$ Bilateral vestibulopathy $\cdot$ Oscillopsia $\cdot$ Presbyvestibulopathy $\cdot$ Stabilization

\section{Introduction}

The vestibular system has two sets of peripheral organs that detect head accelerations and tilt. One of their main functions is to facilitate gaze stabilization. This is made possible to a large extent by a reflex from the vestibular organs to the eyes, called the vestibulo-ocular reflex (VOR) [1]. The VOR generates eye movements in the opposite direction of the head movements, stabilizing the eyes in space during

D. Starkov

dmitrii.n.starkov@gmail.com

Extended author information available on the last page of the article head movements. As a result, the image of the environment remains stable on the retina while in movement.

In bilateral vestibulopathy (BVP), the vestibular function is reduced or absent on both sides [2]. This is a heterogeneous disorder in which the VOR, among other vestibular functions, is impaired [3]. It can lead to insufficient gaze stabilization, resulting in "blurred" vision during head movements, also known as "oscillopsia". Oscillopsia is, next to imbalance, one of the main symptoms of BVP [2, 4-6]. BVP also has a strong negative impact on quality of life and social participation; many BVP-patients suffer from a constant psychological burden caused by fear of falling, decreased activity levels, and social isolation $[2,4,5]$. 
One way to quantify the functional outcome of gaze stabilization, is to test Dynamic Visual Acuity (DVA). DVA reflects the ability of the eyes to distinguish fine details in static objects during head movements [7]. DVA is often tested by comparing the visual acuity in a static condition (i.e., without head movements) to the visual acuity in a dynamic condition (i.e., with head movements). The loss of visual acuity in dynamic conditions (DVAL) is mostly used as outcome measure. Apart from being a functional outcome of the VOR, it also reflects the function of the visual, oculomotor, and vestibular system. That is why not all BVP-patients suffer from oscillopsia: other systems might compensate for the loss of VOR, such as otolith outputs [8,9], automatic spinal locomotor programs [10], or compensatory walking strategies, e.g., reduction of walking speed or stride length [11]. The DVA can be tested in several ways, varying from passive head movements in an office chair, to walking on a treadmill [12-14]. For the latter, Guinand et al. [12] demonstrated a rise in test sensitivity for BVP with increasing locomotor speed: from $76 \%$ at $2 \mathrm{~km} / \mathrm{h}$ to $97 \%$ when combining 2, 4, and $6 \mathrm{~km} / \mathrm{h}$.

It was previously reported that depending on the test protocol, up to $22 \%$ of BVP-patients were not able to complete the DVA test on a treadmill (drop-out), since they could not walk at the test speeds [12]. This increase in drop-out rate might have important implications when DVA while walking is considered as an outcome measure for therapeutic interventions, such as a vestibular implant [15]. However, age on its own, or in combination with BVP, has not yet been taken into account in previous studies, despite evidence of age related differences in gait variability and stability among healthy adults [16-19]. Furthermore, age might also influence DVA. For example, it has recently been demonstrated that DVA during self-generated side to side head movements remains stable in healthy individuals aged 3-49 years, but starts to decline at the age of 50 [20]. Therefore, age might significantly impact the feasibility of the DVA test on a treadmill. This might be important when investigating DVA in BVP, since BVP is more often seen at older ages: most patients are between 50 and 70 years [21].

The objective of this study was to investigate the effects of BVP and age when testing DVA on a treadmill. For this, DVA's of BVP-patients and healthy subjects were evaluated on a treadmill at different speeds. It was hypothesized that BVP and age could significantly influence the drop-out rate and DVA, which might decrease the feasibility of the test in the BVP population.

\section{Methods}

\section{Participants}

Participants were recruited at a tertiary referral center (Maastricht UMC +) between June 2016 and December
2018. Inclusion criteria for BVP-patients were in accordance with the Diagnostic criteria Consensus document of the Classification Committee of the Bárány Society [22]: a horizontal angular VOR gain on both sides $<0.6$ (angular head velocity $150-300^{\circ} / \mathrm{s}$ ) and/or summated slow phase velocity of nystagmus of less than $6 \%$ on each side during bithermal caloric tests $\left(30\right.$ and $44{ }^{\circ} \mathrm{C}, 300 \mathrm{ml}$ in $30 \mathrm{~s}$ ) and/or a horizontal angular VOR gain $<0.1$ upon sinusoidal stimulation on a rotatory chair $\left(0.1 \mathrm{~Hz}, \mathrm{Vmax}=50^{\circ} / \mathrm{s}\right)$ and $/$ or a phase lead $>68^{\circ}$ (time constant of $<5 \mathrm{~s}$ ). In addition, patients needed to be older than 18 years. Patients unable to stop medication against anxiety or depression 1 week before testing, were excluded from the study, as well as those suffering from peripheral neuropathy of the lower extremities.

Healthy subjects were recruited via posters in the hospital and among families and friends of the researchers. A questionnaire was used to rule out, as much as possible, any deficits or diseases that could influence the vestibular system. It comprised the following topics: previous medical history (including otorhinolaryngological, neurological, ophthalmological); use of any medication; known balance problems, recent neck trauma or dizziness in the past 6 months.

All participants were excluded from the study if they were unable to walk on the treadmill at $2 \mathrm{~km} / \mathrm{h}$ or had a vision of -4.0 Diopter or lower (without correction), in which they could not read the first line of the optotypes on the computer screen. In some cases, BVP-patients were allowed to hold handrails of the treadmill to prevent falling (10 BVPpatients). The use of alcohol or other stimulants was forbidden in the $24 \mathrm{~h}$ before examination.

\section{Evaluating age effect on the vestibular function in BVP-patients}

The potential effect of age on vestibular function in BVPpatients (which could affect drop-out rate and DVA) was assessed using outcomes from two tests: the video Head Impulse Test (vHIT, ICS Impulse, GN Otometrics; Taastrup, Denmark) and the caloric test using bithermal $\left(30^{\circ}\right.$ and $40^{\circ} \mathrm{C}$ ) irrigations of water. vHIT gains were calculated for the leftward and rightward directions in the lateral plane, and for the upward and downward directions in right anterior-left posterior and left anterior-right posterior planes. The sum of bithermal maximum peak slow phase velocities (SPV) of the nystagmus was used as outcome measure of the caloric test, calculated separately for each side.

\section{Testing DVA on a treadmill}

Sloan optotypes (C, D, H, K, N, O, R, S, V and Z) projected on a computer screen were used to test visual acuity. The computer screen was placed at eye level and at $2.8 \mathrm{~m}$ from the subject. A Sloan letter was presented on a computer 
screen (LG 24bk55 24"), using a custom program written in Matlab R2010a (The Mathworks, Natick, MA, USA). The program randomly showed five letters in a single-letter sequence at one logarithm of the Minimum Angle of Resolution ( $\log$ MAR). If a minimum of two out of five letters were correctly recognized, the letter size decreased by 0.1 $\operatorname{logMAR}$ and five new letters were shown, one after another. If less than two out of five letters were recognized, the procedure was stopped.

The visual acuity of all BVP-patients and healthy subjects was tested in two conditions: static and dynamic. Static visual acuity was measured when the subject was standing still on the treadmill (1210 model, SportsArt, Inc., Tainan, Taiwan). Visual acuity in dynamic conditions was measured while walking on the treadmill at different speeds (2, 4, $6 \mathrm{~km} / \mathrm{h}$, non-randomized). The study procedure ended either when all walking speeds were completed, or when subjects could not walk at a higher speed. If subjects were not able to complete the test at a specific walking speed, they were considered as a "drop-out" for that walking speed. To ensure the subject's safety, a safety string was clipped to the subject's waist that was connected to the emergency brake of the treadmill.

\section{Data analysis and statistics}

Visual acuity in static and dynamic conditions was calculated as: $\operatorname{LogMAR}=0.1+\operatorname{LogMAR}{ }_{x}-0.02 y$ [23], where " $x$ " was defined as the last optotype line in which two or more letters were read correctly and " $y$ " was defined as the number of correctly read letters at that line.

DVAL was defined as the difference between visual acuity in the static condition and the dynamic conditions. Note that a negative DVAL indicates poorer vision in the tested dynamic condition compared to the static condition. Descriptive statistics were made for age and DVAL. The independent sample $t$ test was used to compare mean age between groups.

To evaluate whether holding the treadmill handrails affected DVAL, an independent $t$ test was used to assess whether a difference in mean DVAL existed between patients who held the treadmill handrails and patients who did not hold the treadmill handrails. Obtained $p$ values were Bonferroni corrected.

The potential effect of age on vestibular function in BVPpatients was analyzed using linear regression analyses. Each model contained age as an independent variable and the corresponding outcome (gain or SPV) as a dependent variable.

Since drop-out at the speed of 4 and $6 \mathrm{~km} / \mathrm{h}$ was perfectly correlated (e.g., drop-out at $4 \mathrm{~km} / \mathrm{h}$ excluded successful completion of the test at $6 \mathrm{~km} / \mathrm{h}$ ), multilevel logistic regression of drop-out (yes or no) on speed, age and group (BVP-patients or healthy subjects) failed (multicollinearity).
Therefore, a new dependent variable reflecting the missing pattern was formed using the following criteria: pattern 1no drop-out at all speeds, pattern 2-drop-out at $6 \mathrm{~km} / \mathrm{h}$, and pattern 3 -drop-out at 4 and $6 \mathrm{~km} / \mathrm{h}$. Then multinomial logistic regression was performed to determine the dependency of drop-out on group and age.

To analyze the effect of age and speed on DVAL, while accounting for the dependence among measurements of the same participant, a linear-mixed effects model was applied. Initially, age, group, speed, and all their two-way interactions were included as fixed factors. Then, the nonsignificant interactions were removed by backward selection. Finally, age, group, speed and group by speed interaction were left in the model. Pairwise comparisons were made per group to compare DVAL at 2, 4, and $6 \mathrm{~km} / \mathrm{h}$. Pairwise comparisons were also made per speed to compare DVAL in BVP-patients and healthy subjects. The significance level, alpha, was set to 0.05 . In case of multiple comparisons, $p$ values were Bonferroni corrected. Data were analyzed in $\mathrm{R}$ (v.3.5.2) and SPSS (v.25).

\section{Ethical considerations}

This study was in accordance with the Declaration of Helsinki (amended version 2013) Approval was obtained by the ethical committees of Maastricht University Medical Centre (NL52768.068.15/METC 151027). All participants provided written informed consent prior to the study.

\section{Results}

\section{Participants}

Forty-four BVP-patients (20 male, mean age 59 years, standard deviation 11 years) and 63 healthy subjects ( 27 male, mean age 46 years, standard deviation 20 years) were included in this study. Although mean age was significantly higher in BVP-patients $(p<0.001)$, ages of $60 \%$ of the healthy subjects were equally distributed within the age range of the tested BVP-patients (41-83 years). Age characteristics of both groups are presented in Table 1. Etiologies of BVP comprised: gentamicin treatment $(n=5)$, vancomycin $(n=1)$, amikacin $(n=1)$, chemotherapy $(n=1)$, Herpes infection $(n=1)$, meningitis $(n=3)$, Hashimoto's disease $(n=1)$, renal failure $(n=1)$, Meniere's Disease $(n=4)$, sequential acute unilateral vestibulopathy $(n=1)$, and genetic $(n=5)$. The etiology remained idiopathic in 20 subjects.

In BVP-patients no significant age effect was found on the outcomes of the vHIT and caloric test $(p \geq 0.161)$. 
Table 1 Age characteristics of the tested groups. The number of participants is shown per age group for both BVP-patients and healthy subjects

\begin{tabular}{lcccccc}
\hline & \multicolumn{2}{l}{ Age group (years) } \\
\cline { 2 - 7 } & $19-30$ & $31-40$ & $41-50$ & $51-60$ & $61-70$ & $71-83$ \\
\hline BVP-patients & 1 & 0 & 9 & 14 & 16 & 4 \\
Healthy subjects & 23 & 2 & 10 & 10 & 10 & 8 \\
\hline
\end{tabular}

\section{Drop-out}

Not all BVP-patients and healthy subjects were able to complete the DVA test at each walking speed, which resulted in dropping-out. The drop-out rate per group, walking speed, and age decade is presented in Fig. 1. Age significantly increased the odds of dropping-out at $6 \mathrm{~km} / \mathrm{h}$ (odds ratio $=1.12, p<0.001$ ), and both age and BVP increased the odds of dropping-out at $4 \mathrm{~km} / \mathrm{h}$, and consequently at $6 \mathrm{~km} / \mathrm{h}$ (Age: odds ratio $=1.15, p=0.008 ;$ BVP: odds ratio $=12.40$, $p=0.038)$.

\section{Dynamic visual acuity loss}

DVAL was significantly lower in BVP-patients at all walking speeds $(p<0.001)$ (Fig. 2). Neither age $(p=0.399)$ nor speed $(p \geq 0.258)$ had a significant effect on DVAL in the group of healthy subjects. In the group of BVP-patients only speed $(p \leq 0.036)$ significantly influenced DVAL: it decreased with an increase of speed. There were no significant differences in DVAL across speeds in patients who did and did not hold the treadmill handrails $(p \geq 0.29)$.

\section{Discussion}

This study investigated the drop-out rate and DVA of BVPpatients and healthy subjects. BVP and age were hypothesized to significantly influence the drop-out rate and DVA obtained in both groups. It was demonstrated that both BVP and age, significantly increased the drop-out rate in both groups at the speed of $4 \mathrm{~km} / \mathrm{h}$ or higher. Regarding DVA, only BVP (not age), significantly decreased DVA in the subjects who were able to walk on the treadmill. Furthermore, DVA significantly decreased with higher walking speeds only in BVP patients. To our knowledge, this study has the largest group of BVP patients in which the influence of BVP and age on the drop-out rate and DVA was tested on a treadmill $[12,14]$.

A higher drop-out rate in both BVP patients and healthy subjects with increasing age and walking speed could be explained by an age-related multisensory decline of the systems involved in maintaining posture and gait [24-26]. In the group of healthy subjects, this might also include presbyvestibulopathy: an age-related decline of the vestibular function [27]. Since BVP mostly occurs at older ages, and BVP-patients are often unable to walk at speeds higher than

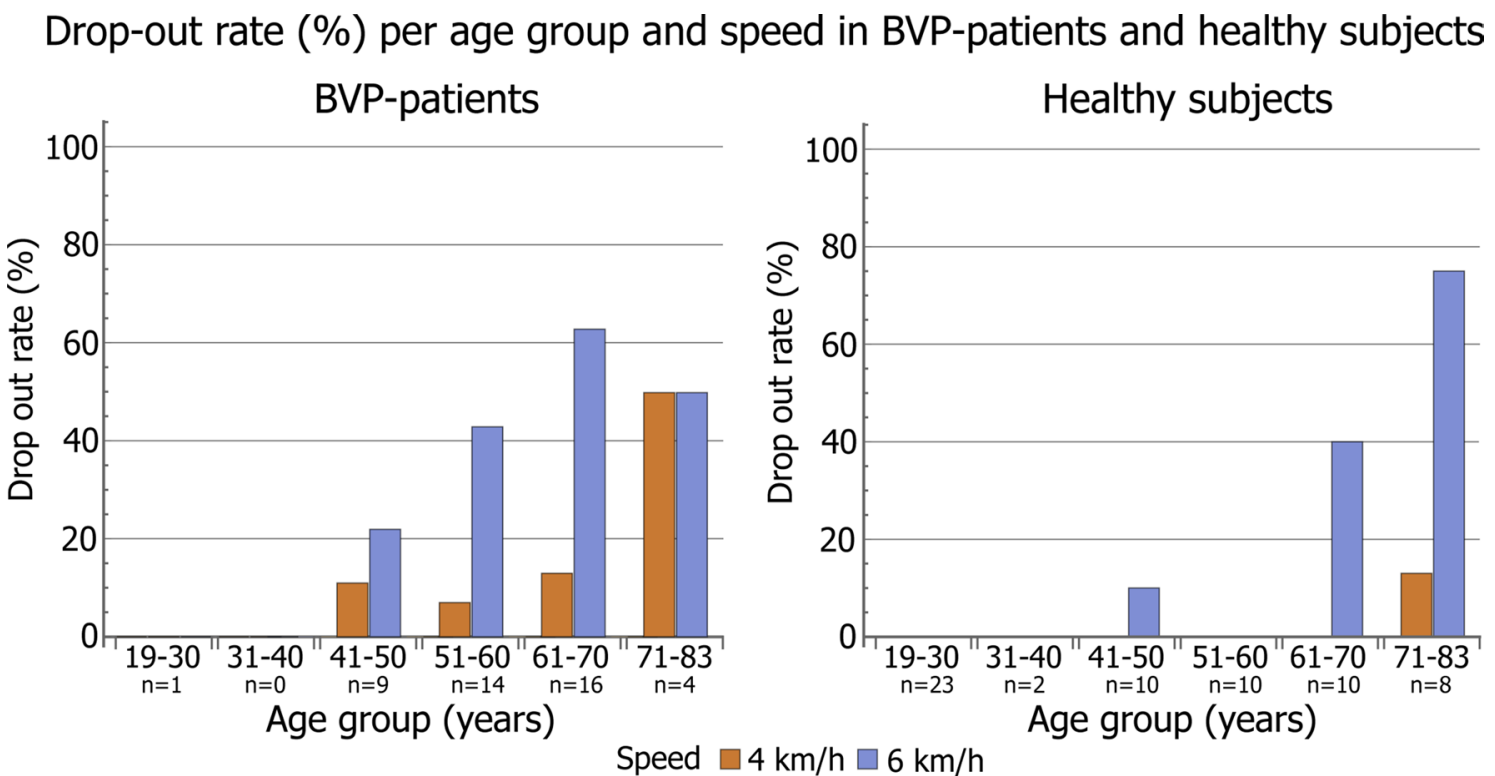

Fig. 1 Drop-out rate (\%) in BVP-patients (left) and healthy subjects (right) per age group and speed 


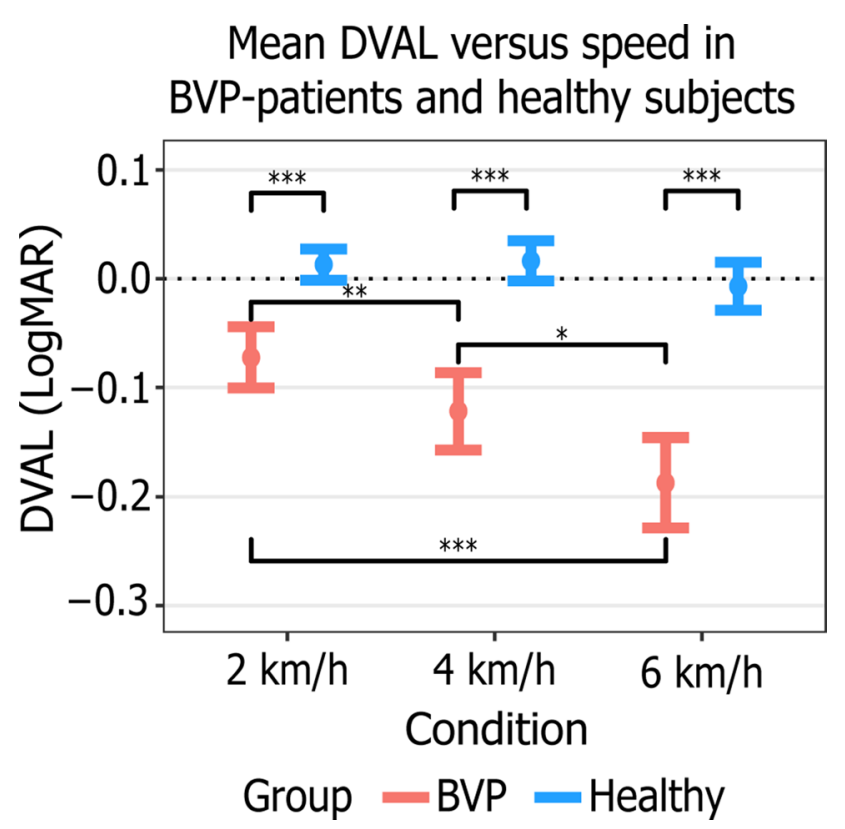

Fig. 2 Mean DVAL versus speed in BVP-patients (red) and healthy subjects (blue). DVAL was calculated as the decline in logMAR between $\mathrm{VA}_{\text {static }}$ and $\mathrm{VA}_{\text {dynamic }}$. Therefore, a negative DVAL value indicates poorer vision while walking on the treadmill, compared to standing still. Error bars represent 95\% confidence interval. Three asterisks $(* * *)$ indicate $p<0.001$, two asterisks $(* *)$ indicate $p<0.01$, one asterisk $(*)$ indicates $p<0.05$

$5 \mathrm{~km} / \mathrm{h}[21,26,28]$, it might be questioned whether testing DVA at fixed speeds on a treadmill would be a reasonable outcome measure for vestibular rehabilitation of BVPpatients in a research setting. However, testing DVA while walking is only one of the few "close to reality" functional outcome measures of vestibular function in the vertical plane [12]. It could, therefore, be proposed to individually adjust the walking speed for each patient, using their "preferred" walking speed. In older subjects on group level, the preferred walking speed range would probably be between $2 \mathrm{~km} / \mathrm{h}$ and (below) $6 \mathrm{~km} / \mathrm{h}$. After all, mean DVA is already significantly reduced at $2 \mathrm{~km} / \mathrm{h}$ [12], the self-selected walking speed for patients with vestibular dysfunction is about $3 \mathrm{~km} / \mathrm{h}[29$, 30 ], and at $6 \mathrm{~km} / \mathrm{h}$ both healthy subjects and BVP patients above 40 years show a significantly increased drop-out rate. The use of a preferred walking speed was already successfully demonstrated in BVP-patients fitted with a prototype vestibular implant [31]. Nevertheless, other possible functional outcome measures could also be hypothesized which do not involve walking. For instance, testing DVA with actively generated head movements while sitting on a chair [20] or the functional head impulse test [32]. However, it should be taken into account that each specific way of testing DVA examines (to a certain extent) different parts, sensitivities and mechanisms of the vestibular system: e.g., the semicircular canals, otolith organs, low- and high-frequency sensitivity, and compensatory strategies. This has already been revealed by the lack of correlation between outcomes of the DVA test on a treadmill, and the functional head impulse test [33].

Conflicting evidence exists regarding the effect of age on DVA when tested on a treadmill in healthy subjects. One previous study described a significant age effect on DVA at only $4 \mathrm{~km} / \mathrm{h}$ (not at 3,6 , and $9 \mathrm{~km} / \mathrm{h}$ ) [14], while another study found a significant age effect at 4 and $6 \mathrm{~km} / \mathrm{h} \mathrm{[12].} \mathrm{The}$ present study did not find any effect of age on DVA. This might partially be explained by different inclusion criteria used for the group of healthy subjects; Verbecque et al. [14] used a questionnaire and age-specific static balance testing, Guinand et al. [12] used the Video Head Impulse Test, and this study used a questionnaire to rule out (as much as possible) any deficits or diseases that could influence the vestibular system. Furthermore, the different statistical analyses and testing paradigms, including the used optotype charts and DVA cut-off values, could contribute to the conflicting evidence. Nevertheless, the findings of this study do not rule out any age effect, since the DVA in the group of subjects who dropped-out, remains unknown. Furthermore, DVA tested during self-generated side to side head movements significantly declines from the age of 50 [20]. Therefore, taking the drop-out rate and the evidence regarding age effect into account, it would be recommended to use agematched controls when testing DVA in research settings and to limit maximum walking speed for older subjects (e.g., below $6 \mathrm{~km} / \mathrm{h}$ ).

BVP-patients showed, on group level, worse DVA than healthy subjects at all walking speeds. This finding is congruent with previously described results [12]. In addition to impaired vestibular function, this DVA decrease might (partially) be induced by attention deficits [34] or the inability to correctly perform dual-tasks, which can be present in BVPpatients [35]. However, DVA overlapped between BVPpatients and age-matched controls. This can be explained by multiple factors. First, the DVA is a functional outcome of a multisensory system. Input from the visual, vestibular and oculomotor systems is centrally processed, which facilitates adaptation and compensation mechanisms. An example of such a compensation mechanism is minimizing head movements to improve gaze stabilization. Second, DVA can be trained. It has been shown that vestibular rehabilitation exercises facilitate the recovery of gaze during head movements in BVP-patients [36]. Furthermore, in patients with unilateral peripheral vestibulopathy, covert saccades can improve DVA [37,38]. Third, during the DVA test on a treadmill, (partially) active movements are made. These active movements are less useful in discriminating between BVP-patients and healthy subjects, as compared to passive movements $[13,39]$. In addition, during stereotyped locomotion, feed-forward signals from an efference copy of 
the locomotor commands suppress the VOR in the vertical plane, which can mediate gaze stabilization [40]. Finally, walking speed affects gait parameters in BVP-patients [26]. All these factors imply that the DVA test on a treadmill should mainly be used to evaluate the functional status of the vestibular system.

\section{Limitations of the study}

In contrast to previous studies $[12,14]$ only a questionnaire was used to determine whether a subject was healthy or not. None of the vestibular tests like the Video Head Impulse Test or caloric test were performed in healthy subjects. It cannot be ruled out that results of the healthy subjects might have been influenced by factors like presbyvestibulopathy or asymptomatic vestibulopathies. This could mainly imply that the effects of BVP on drop-out and DVA found in this study, might be underestimated. Height of the participants was not measured, which could affect stride length and, therefore, head movement amplitude and, consequently, DVA. Furthermore, statistical power in the highest age groups was small due to the high drop-out rate. It can, therefore, only be stated that DVA was not significantly influenced by age in BVP-patients and healthy subjects who were able to walk at the tested speeds. The DVA of subjects who were unable to walk could not be determined using the treadmill test.

\section{Conclusion}

DVA tested while walking on a treadmill, is one of the few "close to reality" functional outcome measures of vestibular function in the vertical plane. It is able to demonstrate significant loss of DVA in bilateral vestibulopathy patients. However, since bilateral vestibulopathy and age significantly increase the drop-out rate at faster walking speeds, it is recommended to use age-matched controls. Furthermore, it could be considered to use an individual "preferred" walking speed and to limit maximum walking speed in older subjects when testing DVA on a treadmill.

\section{Acknowledgements Not applicable.}

Author contributions All authors participated in the design of the experimental protocol and analysis. FL, MS and DS carried out the experiments. AMLJ and MP performed the statistical analysis. DS, MS and RvdB wrote the manuscript, and all authors contributed to its editing. RvdB, AP, NG, VR, NH, AH, CM, KM, and LV critically revised the manuscript. RvdB, AP, NG and HK supervised all aspects of the work.

Funding Not applicable.

Data availability Data is available on the reasonable request to the corresponding author.

\section{Compliance with ethical standards}

Conflicts of interest The authors declare that they have no conflict of interest. Med-El has provided funding for research and travel for RvdB, AP, NG and HK. DS, MP, RvdB and HK were supported by The Tomsk State University competitiveness improvement programme.

Ethics approval This study was in accordance with the Declaration of Helsinki (amended version 2013) Approval was obtained by the ethical committees of Maastricht University Medical Centre (NL52768.068.15 / METC 151027).

Consent to participate All participants provided informed consent for participation prior to the study.

Consent for publication All participants provided informed consent for publication prior to the study.

Code availability Not applicable.

Open Access This article is licensed under a Creative Commons Attribution 4.0 International License, which permits use, sharing, adaptation, distribution and reproduction in any medium or format, as long as you give appropriate credit to the original author(s) and the source, provide a link to the Creative Commons licence, and indicate if changes were made. The images or other third party material in this article are included in the article's Creative Commons licence, unless indicated otherwise in a credit line to the material. If material is not included in the article's Creative Commons licence and your intended use is not permitted by statutory regulation or exceeds the permitted use, you will need to obtain permission directly from the copyright holder. To view a copy of this licence, visit http://creativecommons.org/licenses/by/4.0/.

\section{References}

1. Fetter M (2007) Vestibulo-ocular reflex. Neuro-ophthalmology. KARGER, Basel, pp 35-51

2. Hain T, Cherchi M, Yacovino D (2013) Bilateral vestibular loss. Semin Neurol 33:195-203. https://doi.org/10.1055/s-0033-13545 97

3. van de Berg R, Guinand N, Stokroos RJ et al (2011) The vestibular implant: Quo vadis? Front Neurol 1:14. https://doi.org/10.3389/ fneur.2011.00047

4. Tilikete C, Vighetto A (2011) Oscillopsia: causes and management. Curr Opin Neurol 24:38-43. https://doi.org/10.1097/ WCO.0b013e328341e3b5

5. van de Berg R, van Tilburg M, Kingma H (2015) Bilateral vestibular hypofunction: challenges in establishing the diagnosis in adults. ORL J Otorhinolaryngol Relat Spec 77:197-218. https:// doi.org/10.1159/000433549

6. Hermann R, Ionescu EC, Dumas O et al (2018) Bilateral vestibulopathy: vestibular function, dynamic visual acuity and functional impact. Front Neurol 9:555. https://doi.org/10.3389/fneur .2018 .00555

7. Palidis DJ, Wyder-Hodge PA, Fooken J, Spering M (2017) Distinct eye movement patterns enhance dynamic visual acuity. PLoS ONE 12:e0172061. https://doi.org/10.1371/journal.pone.0172061

8. Demer JL, Honrubia V, Baloh RW (1994) Dynamic visual acuity: a test for oscillopsia and vestibulo-ocular reflex function. Am J Otol 15:340-347

9. McGath JH, Barber HO, Stoyanoff S (1989) Bilateral vestibular loss and oscillopsia. J Otolaryngol 18:218-221 
10. Brandt T, Strupp M, Benson J (1999) You are better off running than walking with acute vestibulopathy. Lancet 354:746. https:// doi.org/10.1016/S0140-6736(99)03179-7

11. Schniepp R, Schlick C, Schenkel F et al (2017) Clinical and neurophysiological risk factors for falls in patients with bilateral vestibulopathy. J Neurol 264:277-283. https://doi.org/10.1007/s0041 5-016-8342-6

12. Guinand N, Pijnenburg M, Janssen M, Kingma H (2012) Visual acuity while walking and oscillopsia severity in healthy subjects and patients with unilateral and bilateral vestibular function loss. Arch Otolaryngol Neck Surg 138:301. https://doi.org/10.1001/ archoto. 2012.4

13. Tian J, Shubayev I, Demer J (2002) Dynamic visual acuity during passive and self-generated transient head rotation in normal and unilaterally vestibulopathic humans. Exp Brain Res 142:486-495. https://doi.org/10.1007/s00221-001-0959-7

14. Verbecque E, Van Criekinge T, Vanloot D et al (2018) Dynamic visual acuity test while walking or running on treadmill: reliability and normative data. Gait Posture 65:137-142. https://doi. org/10.1016/j.gaitpost.2018.07.166

15. Guinand N, Van de Berg R, Cavuscens S et al (2017) The video head impulse test to assess the efficacy of vestibular implants in humans. Front Neurol 8:600. https://doi.org/10.3389/fneur .2017 .00600

16. Kang HG, Dingwell JB (2008) Separating the effects of age and walking speed on gait variability. Gait Posture 27:572-577. https ://doi.org/10.1016/j.gaitpost.2007.07.009

17. Menz HB, Lord SR, Fitzpatrick RC (2003) Age-related differences in walking stability. Age Ageing 32:137-142. https://doi. org/10.1093/ageing/32.2.137

18. Terrier P, Reynard F (2015) Effect of age on the variability and stability of gait: a cross-sectional treadmill study in healthy individuals between 20 and 69 years of age. Gait Posture 41:170-174. https://doi.org/10.1016/j.gaitpost.2014.09.024

19. McCrum C, Karamanidis K, Grevendonk L et al (2020) Older adults demonstrate interlimb transfer of reactive gait adaptations to repeated unpredictable gait perturbations. GeroScience 42:39 49. https://doi.org/10.1007/s11357-019-00130-x

20. Li C, Beaumont JL, Rine RM et al (2014) Normative scores for the NIH toolbox dynamic visual acuity test from 3 to 85 years. Front Neurol 5:223. https://doi.org/10.3389/fneur.2014.00223

21. Schubert MC, Tusa RJ, Grine LE, Herdman SJ (2004) Optimizing the sensitivity of the head thrust test for identifying vestibular hypofunction. Phys Ther 84:151-158

22. Strupp M, Kim J-S, Murofushi T et al (2017) Bilateral vestibulopathy: diagnostic criteria consensus document of the classification committee of the Bárány society. J Vestib Res 27:177-189. https://doi.org/10.3233/VES-170619

23. Carlson NB, Kurtz D (2015) Clinical procedures for ocular examination, 4th edn. McGraw-Hill Education, New York

24. Lord SR, Delbaere K, Sturnieks DL (2018) Aging. Handbook of clinical neurology. Elsevier, New York, pp 157-171

25. Lord SR, Stephen R, Sherrington C, Menz HB (2001) Falls in older people: risk factors and strategies for prevention. Cambridge University Press, Cambridge

26. McCrum C, Lucieer F, van de Berg R et al (2019) The walking speed-dependency of gait variability in bilateral vestibulopathy and its association with clinical tests of vestibular function. Sci Rep 9:1-12. https://doi.org/10.1038/s41598-019-54605-0

27. Agrawal Y, Van De Berg R, Wuyts F et al (2019) Presbyvestibulopathy: diagnostic criteria consensus document of the classification committee of the Bárány Society. J Vestib Res Equilib Orientat 29:161-170. https://doi.org/10.3233/VES-190672

28. Lambert S, Sigrist A, Delaspre O et al (2010) Measurement of dynamic visual acuity in patients with vestibular areflexia. Acta Otolaryngol 130:820-823. https://doi.org/10.3109/0001648090 3426592

29. Schmidheiny A, Swanenburg J, Straumann D et al (2015) Discriminant validity and test re-test reproducibility of a gait assessment in patients with vestibular dysfunction. BMC Ear Nose Throat Disord. https://doi.org/10.1186/s12901-015-0019-8

30. Kim SC, Kim JY, Lee HN et al (2014) A quantitative analysis of gait patterns in vestibular neuritis patients using gyroscope sensor and a continuous walking protocol. J Neuroeng Rehabil 11:58. https://doi.org/10.1186/1743-0003-11-58

31. Starkov D, Guinand N, Lucieer F et al (2019) Restoring the highfrequency dynamic visual acuity with a vestibular implant prototype in humans. Audiol Neurotol. https://doi.org/10.1159/00050 3677

32. Colagiorgio P, Colnaghi S, Versino M, Ramat S (2013) A new tool for investigating the functional testing of the VOR. Front Neurol 4:165. https://doi.org/10.3389/fneur.2013.00165

33. van Dooren TS, Lucieer FMP, Duijn S et al (2019) The functional head impulse test to assess oscillopsia in bilateral vestibulopathy. Front Neurol 10:365. https://doi.org/10.3389/fneur.2019.00365

34. Dobbels B, Mertens G, Gilles A et al (2019) Cognitive function in acquired bilateral vestibulopathy: a cross-sectional study on cognition, hearing, and vestibular loss. Front Neurosci 13:340. https://doi.org/10.3389/fnins.2019.00340

35. Danneels M, Van Hecke R, Keppler H et al (2019) Psychometric properties of cognitive-motor dual-task studies with the aim of developing a test protocol for persons with vestibular disorders: a systematic review. Ear Hear. https://doi.org/10.1097/AUD.00000 00000000748

36. Herdman SJ, Hall CD, Schubert MC et al (2007) Recovery of dynamic visual acuity in bilateral vestibular hypofunction. Arch Otolaryngol Neck Surg 133:383. https://doi.org/10.1001/archo tol.133.4.383

37. Wettstein VG, Weber KP, Bockisch CJ, Hegemann SC (2016) Compensatory saccades in head impulse testing influence the dynamic visual acuity of patients with unilateral peripheral vestibulopathy. J Vestib Res 26:395-402

38. Halmagyi GM, Curthoys IS (1988) A clinical sign of canal paresis. Arch Neurol 45:737-739. https://doi.org/10.1001/archn eur.1988.00520310043015

39. Vital D, Hegemann SCA, Straumann D et al (2010) A new dynamic visual acuity test to assess peripheral vestibular function. Arch Otolaryngol Neck Surg 136:686. https://doi.org/10.1001/ archoto. 2010.99

40. Dietrich H, Wuehr M (2019) Selective suppression of the vestibulo-ocular reflex during human locomotion. J Neurol. https://doi. org/10.1007/s00415-019-09352-7 


\section{Affiliations}

\section{Starkov ${ }^{1,2,11}$ (D) M. Snelders ${ }^{1} \cdot$ F. Lucieer ${ }^{1}$ - A. M. L. Janssen ${ }^{1,3} \cdot$ M. Pleshkov ${ }^{1,2} \cdot$ H. Kingma ${ }^{1,2} \cdot$ V. van Rompaey ${ }^{4,5}$. N. Herssens $s^{6,7} \cdot$ A. Hallemans $s^{6,7,8} \cdot$ L. Vereeck $^{6,7} \cdot$ C. McCrum $^{9} \cdot$ K. Meijer ${ }^{9} \cdot$ N. Guinand ${ }^{1,10}$ - A. Perez-Fornos ${ }^{10}$. R. van de Berg ${ }^{1,2}$}

1 Division of Balance Disorders, Department of Otorhinolaryngology and Head and Neck Surgery, Maastricht University Medical Centre, Maastricht, The Netherlands

2 Faculty of Physics, Tomsk State Research University, Tomsk, Russia

3 Department of Methodology and Statistics, Care and Public Health Research Institute (CAPHRI), Maastricht University, Maastricht, The Netherlands

4 Faculty of Medicine and Health Sciences, University of Antwerp, Antwerp, Belgium

5 Department of Otorhinolaryngology and Head and Neck Surgery, Antwerp University Hospital, Edegem, Belgium

6 Department of Rehabilitation Sciences and Physiotherapy, Faculty of Medicine and Health Science, University of Antwerp, Antwerp, Belgium
7 Multidisciplinary Motor Centre Antwerp (M2OCEAN), University of Antwerp, Antwerp, Belgium

8 The Research Group MOVANT (MOVement ANTwerp), University of Antwerp, Antwerp, Belgium

9 Department of Nutrition and Movement Sciences, NUTRIM School of Nutrition and Translational Research in Metabolism, Maastricht University Medical Centre+, Maastricht, The Netherlands

10 Service of Otorhinolaryngology Head and Neck Surgery, Department of Clinical Neurosciences, Geneva University Hospitals, Geneva, Switzerland

11 Maastricht University ENT Department, P. Debyelaan 25, 6229 HX Maastricht, The Netherlands 